\title{
Enfrentamento da Covid-19 nas unidades de assistência farmacêutica na Secretaria de Saúde do Paraná
}

\author{
Emergency response actions to Covid-19 from a \\ pharmaceutical assistance perspective in the Para- \\ ná Secretary of Health
}

Paula Rossignoli ${ }^{1}$, Deise Regina Sprada Pontarolli², Claudia Boscheco Moretoni ${ }^{3}$, Priscilla Marys Limberger', Margely Nunes ${ }^{5}$

1. ORCID: https://orcid.org/0000-0002-8691-7359. Farmacêutica da Secretaria de Estado da Saúde do Paraná, Curitiba/PR, Brasil. Mestre em Ciências Farmacêuticas pela Universidade Federal do Paraná.

2. ORCID: https://orcid.org/0000-0002-0694-6740. Farmacêutica da Secretaria de Estado da Saúde do Paraná, Curitiba/PR, Brasil. Especialista em Gestão da Assistência Farmacêutica do SUS pela Escola Nacional de Saúde Pública.

3. ORCID: https://orcid.org/0000-0002-3924-7348. Farmacêutica da Secretaria de Estado da Saúde do Paraná, Curitiba/PR, Brasil. Mestre em Ciências Farmacêuticas pela Universidade Federal do Paraná.

4. ORCID: https://orcid.org/0000-0002-2788-1368. Farmacêutica da Secretaria de Estado da Saúde do Paraná, Curitiba/PR, Brasil. Mestranda em Assistência Farmacêutica pela Universidade Federal do Paraná.

5. ORCID: https://orcid.org/0000-0001-7172-5429. Farmacêutica da Secretaria de Estado da Saúde do Paraná, Curitiba/PR, Brasil. Mestre em Ciências Farmacêuticas pela Universidade Federal do Paraná.

CONTATO: Paula Rossignoli | Endereço: Rua Piquiri, 170. Curitiba/PR | Telefone: 4133304447 | E-mail: paula.rossignoli@sesa.pr.gov.br

RESUMO A chegada da epidemia de Covid-19 ao Paraná tornou necessária a adoção de medidas pela Assistência Farmacêutica da Secretaria de Estado da Saúde que reduzisse a circulação de pessoas e reorientasse os trabalhos nas farmácias das Regionais de Saúde. Cerca de 112 mil usuários são atendidos nessas farmácias para recebimento de medicamentos do Componente Especializado e elenco complementar. Dentre as estratégias para diminuição de circulação de usuários estão entrega antecipada de medicamentos, reno- 
vações automáticas do tratamento, ferramenta para cadastro online de novos usuários e ampliação do serviço de entrega em casa. A entrega antecipada de medicamentos, o cadastro online e a entrega domiciliar proporcionaram juntos uma redução da presença de cerca de 80 mil usuários nas farmácias das RS entre março e julho de 2020. As ações adotadas foram efetivas na redução da circulação de pessoas protegendo usuários e equipe da AF.

DESCRITORES: Assistência Farmacêutica. Covid-19. Sistema Único de Saúde.

\begin{abstract}
The arrival of the Covid-19 epidemic in Paraná required the Pharmaceutical Assistance Department (AF) of the Paraná Secretary of Health to adopt measures to reduce people's circulation and redirect the efforts of the pharmacies of all Regional Health Offices (RS). About 112 thousand users count on these pharmacies to receive medicines included in the Specialized Component and Complementary medicines list. Among the strategies to reduce the circulation of users are early delivery of medicines; automated renewals of treatment; a tool for online registration of new users and expansion of the home delivery service. The early delivery of medicines, online registration and home delivery together made it possible for 80 thousand users not to have to physically visit RS pharmacies between March 2020 and July of the same year, protecting not only the users themselves but also AF people.
\end{abstract}

DESCRIPTORS: Pharmaceutical Assistance. Covid-19. Unified Health System.

\title{
INTRODUÇÃO
}

E m 30 de janeiro de 2020 a Organização Mundial da Saúde (OMS) declarou o surto da doença causada pelo novo coronavírus (Covid-19) como uma Emergência de Saúde Pública de Importância Internacional - o mais alto nível de alerta da Organização, conforme previsto no Regulamento Sanitário Internacional’1.

O Ministério da Saúde brasileiro estabeleceu, em 3 de fevereiro, "Emergência em Saúde Pública de Importância Nacional" em decorrência da infecção humana pelo novo coronavírus ${ }^{2}$, e adotou medidas visando a proteção da coletividade em todo o território nacional ${ }^{3}$. O primeiro caso de Covid-19 no Brasil foi confirmado em 26 de fevereiro, no município de São Paulo4. Nesta data, o mundo registrava 81.109 casos confirmados 5 . Em 11 de março a Covid-19 foi caracterizada como 
uma pandemia pela OMS, com o reconhecimento de surtos da doença em vários países e regiões do mundo ${ }^{1}$. Nesse mesmo dia o primeiro caso foi confirmado no Estado do Paranáa-7.

O Governo do Estado do Paraná publicou, em 16 de março, o Decreto $n^{\circ}$ 4.230/2020, regulamentando a organização de medidas estaduais para enfrentamento da emergência de saúde pública de importância internacional decorrente do Coronavírus - Covid-19. A avaliação do cenário epidemiológico do Estado do Paraná em relação à infecção por esta patologia demonstrou expansão no número de casos, motivando a declaração de situação de emergência em todo o território paranaense em 19 de março ${ }^{9}$. Nessa data, a OMS já computava 209.839 casos confirmados no mundo, com 8.778 óbitos $^{10}$. O Brasil contava com 291 casos confirmados e um óbito ${ }^{10}$ e o Paraná, por sua vez, 23 casos confirmados ${ }^{11}$. Em 23 de março o Governo do Estado do Paraná declara, por meio do Decreto n 4.319/2020, estado de calamidade pública, que perdura até o presente momento ${ }^{12}$. Em 28 de julho são 16.341 .920 casos confirmados e 650.805 óbitos no mundo ${ }^{13}$. O Brasil conta com 2.419.091 casos confirmados e 87.004 óbitos $^{13}$. No Paraná, somam-se 68.644 casos confirmados e 1.721 óbitos nessa mesma data ${ }^{14}$.

\section{Assistência Farmacêutica na Secretaria de Estado da Saúde do Paraná}

A Secretaria de Estado da Saúde do Paraná - SESA/PR está organizada em 22 Regionais de Saúde (RS). Cada Regional conta com uma farmácia e uma Central de Abastecimento Farmacêutico (CAF). As farmácias são responsáveis pela gestão regional do Componente Especializado da Assistência Farmacêutica (CEAF), e realizam a dispensação dos medicamentos desse componente e do elenco complementar da SESA/PR. As CAF, por sua vez, são responsáveis pelo recebimento, armazenamento, programação e distribuição dos medicamentos de todos os Componentes da Assistência Farmacêutica (Básico, Estratégico e Especializado) aos municípios de sua área de abrangência.

O CEAF é uma estratégia de acesso a medicamentos, no âmbito do Sistema Único de Saúde (SUS), caracterizado pela busca da garantia da integralidade do trata- 
mento medicamentoso em nível ambulatorial ${ }^{15}$. Está contemplado no CEAF o atendimento dos usuários com condições clínicas ou doenças que requerem um tratamento mais complexo, geralmente com medicamentos mais caros e diagnóstico realizado por especialistas, sendo os critérios definidos pelo Ministério da Saúde por meio dos Protocolos Clínicos e Diretrizes Terapêuticas (PCDT). Este Componente é regulamentado pelas Portarias de Consolidação $n^{\circ} 2$ e $n^{\circ} 6$, de 28 de setembro de 2017 16 -17. A execução do CEAF é de responsabilidade das Secretarias de Saúde dos Estados e do Distrito Federal e envolve as etapas de solicitação, avaliação, autorização, dispensação e renovação da continuidade do tratamento.

No Paraná, para a solicitação dos medicamentos do CEAF, o usuário deve se dirigir à Farmácia da Regional de Saúde ou até a farmácia do seu município de residência, nos casos em que o município assumiu a dispensação deste Componente, e apresentar os documentos definidos na Portaria de Consolidação n 2/2017 e nos PCDT. A solicitação é submetida à avaliação técnica (de caráter documental) e à autorização (de caráter administrativo), que aprova ou não o fornecimento do medicamento ${ }^{16}$. A dispensação do medicamento ao paciente geralmente é feita para um ou dois meses de tratamento, e a cada trimestre o usuário deve renovar a continuidade do tratamento, mediante apresentação de documentos previstos nos PCDT para o monitoramento do tratamento ${ }^{16}$. Os medicamentos do CEAF são adquiridos pela SESA/PR por meio do Centro de Medicamentos do Paraná - CEMEPAR, órgão central de operações logísticas do Estado, e pelo Ministério da Saúde. O CEMEPAR é responsável pelo recebimento e distribuição mensal de todos os medicamentos às $22 \mathrm{RS}^{18}$.

No primeiro semestre de 2020, foram distribuídos às Regionais de Saúde cerca de 140 mil unidades de medicamentos do CEAF e elenco complementar da SESA/ PR, correspondendo a 250 diferentes apresentações de medicamentos, a um custo aproximado de 670 milhões de reais, de acordo com dados extraídos do Sysmed

O acesso a esse grupo de medicamentos tem sido crescente no Paraná, não

1. Sysmed é o sistema de informação para o gerenciamento do estoque de medicamentos no Cemepar. 
somente devido à incorporação de novas tecnologias, mas principalmente à estrutura organizada das farmácias e centrais de abastecimento farmacêutico das Regionais de Saúde, sustentada por um robusto sistema de informação gerencial, o Sismedex 2 .

Em julho de 2020, cerca de 260 mil usuários estavam cadastrados junto à SESA/ PR para recebimento desse grupo de medicamentos, conforme dados extraídos do Sismedex. Desse total de usuários, $44 \%$ são atendidos diretamente nas farmácias das Regionais de Saúde. Os demais são atendidos em farmácias municipais ou outros serviços de saúde.

Dado esse cenário, este trabalho tem por objetivo apresentar o relato de experiência do planejamento, da execução e dos resultados das ações adotadas pela Assistência Farmacêutica da SESA/PR voltadas à redução do potencial de contaminação de usuários e servidores pelo novo coronavírus, no âmbito das farmácias e centrais de abastecimento farmacêutico das Regionais de Saúde.

\section{MÉTODO}

Trata-se de um trabalho descritivo com relato de experiência acerca das medidas adotadas no âmbito das unidades de Assistência Farmacêutica da SESA/PR no enfrentamento à pandemia de Covid-19. Serão apresentados o planejamento e a execução de ações para diminuir a circulação de pessoas nas farmácias sob gestão da SESA/PR e medidas adotadas para maior proteção dos servidores que atuam nessa área. Também serão apresentados os resultados mensuráveis decorrentes dessas ações.

O planejamento e o acompanhamento das medidas, que abrangeu as 22 Regionais de Saúde, foram realizados pela Coordenação de Assistência Farmacêutica (COAF) e pelo Centro de Medicamentos do Paraná (CEMEPAR), assim que o Decreto $n^{\circ} 4.230 / 2020$ foi publicado em 16 de março.

2. Sismedex é o sistema de informação para a gestão do CEAF no Paraná. 


\section{RESULTADOS E DISCUSSÃO}

As ações de enfrentamento à pandemia foram concentradas em duas áreas: medidas para diminuir a circulação de usuários nas farmácias e orientações às equipes das farmácias e centrais de abastecimento farmacêutico das Regionais de Saúde sobre rotinas e processos de trabalho.

\section{MEDIDAS PARA DIMINUIR A CIRCULAÇÃO DE USUÁRIOS}

As estratégias adotadas para diminuir a circulação de usuários nas farmácias das Regionais de Saúde compreenderam: a) envio de mensagens com orientações aos usuários; b) entrega antecipada de medicamentos para dois ou três meses de tratamento; c) renovações automáticas para continuidade do tratamento; d) desenvolvimento de ferramenta para cadastro online do usuário na farmácia; e e) ampliação do serviço "Remédio em casa”.

\section{a) Envio de mensagens com orientações aos usuários}

Por meio de uma funcionalidade presente no Sismedex, que permite o envio de mensagens aos usuários cadastrados, nos dias 16 e 17 de março foi enviado aos pacientes atendidos nas Farmácias das Regionais de Saúde um SMS (Short Message Service) com a seguinte orientação: "Se tiver febre, tosse ou dificuldade para respirar não venha à farmácia e autorize alguém a pegar seu remédio. Venha apenas uma pessoa no horário agendado".

Apesar de haver agendamento de dia e horário para o atendimento nas farmácias regionais, não é incomum o comparecimento do usuário muito antes da hora marcada. O reforço para comparecer no horário agendado, a restrição de acesso à farmácia antes do horário e da entrada de acompanhantes trouxeram diminuição do número de pessoas no interior das unidades, evitando aglomerações. 


\section{b) Entrega antecipada de medicamentos para 2 ou 3 meses de tratamento}

A dispensação de medicamentos do CEAF nas farmácias é feita para 1 ou 2 meses de tratamento. Dessa forma, parte dos usuários retorna mensalmente às farmácias para o recebimento dos medicamentos.

A partir de 16 de março de 2020, as farmácias das Regionais de Saúde foram orientadas a realizar a dispensação antecipada de medicamentos aos usuários para dois ou três meses de tratamento, de acordo com a disponibilidade do produto em seus estoques. Essa medida teve por objetivo diminuir a frequência de idas do usuário à farmácia, levando à diminuição da circulação de pessoas nesse ambiente.

Além dessa análise feita por cada uma das farmácias para início imediato da entrega antecipada, o CEMEPAR passou a verificar mensalmente o seu estoque para definir quais itens poderiam ser disponibilizados pelas farmácias para dois ou três meses de tratamento. Com essas análises verificou-se que, de um total de 255 medicamentos gerenciados, 140 itens em média poderiam ser disponibilizados para três meses e outros 50 itens, em média, para dois meses de tratamento.

Na Tabela 1 são apresentados os resultados das análises mensais do número de itens de medicamentos com possibilidade de entrega antecipada para dois e para três meses de tratamento.

Tabela 1. Número de itens de medicamentos definido a cada mês com possibilidade de entrega antecipada para dois ou três meses de tratamento.

\begin{tabular}{lcc}
\hline \multicolumn{1}{c}{ MÊS } & $\begin{array}{c}\text { Quantidade de } \\
\text { medicamentos para 2 } \\
\text { meses de tratamento }\end{array}$ & $\begin{array}{c}\text { Quantidade de } \\
\text { medicamentos para 3 } \\
\text { meses de tratamento }\end{array}$ \\
\hline Março/20 & 51 & 165 \\
Abril/20 & 40 & 143 \\
Maio/20 & 46 & 145 \\
Junho/20 & 59 & 121 \\
Julho/20 & 56 & 144 \\
\hline
\end{tabular}

Fonte: Elaborado pelos autores (2020). 
A informação mensal relativa a quais itens poderiam ser entregues de forma antecipada trouxe às farmácias a possibilidade de reorganização das agendas de dispensação, com diminuição importante do número de atendimentos diários.

No Gráfico 1 é possível observar a diminuição no número de pacientes atendidos nas farmácias regionais a partir de abril de 2020.

Gráfico 1. Quantidade mensal de usuários atendidos com dispensação de medicamentos de janeiro a julho/2020 nas farmácias das Regionais de Saúde.

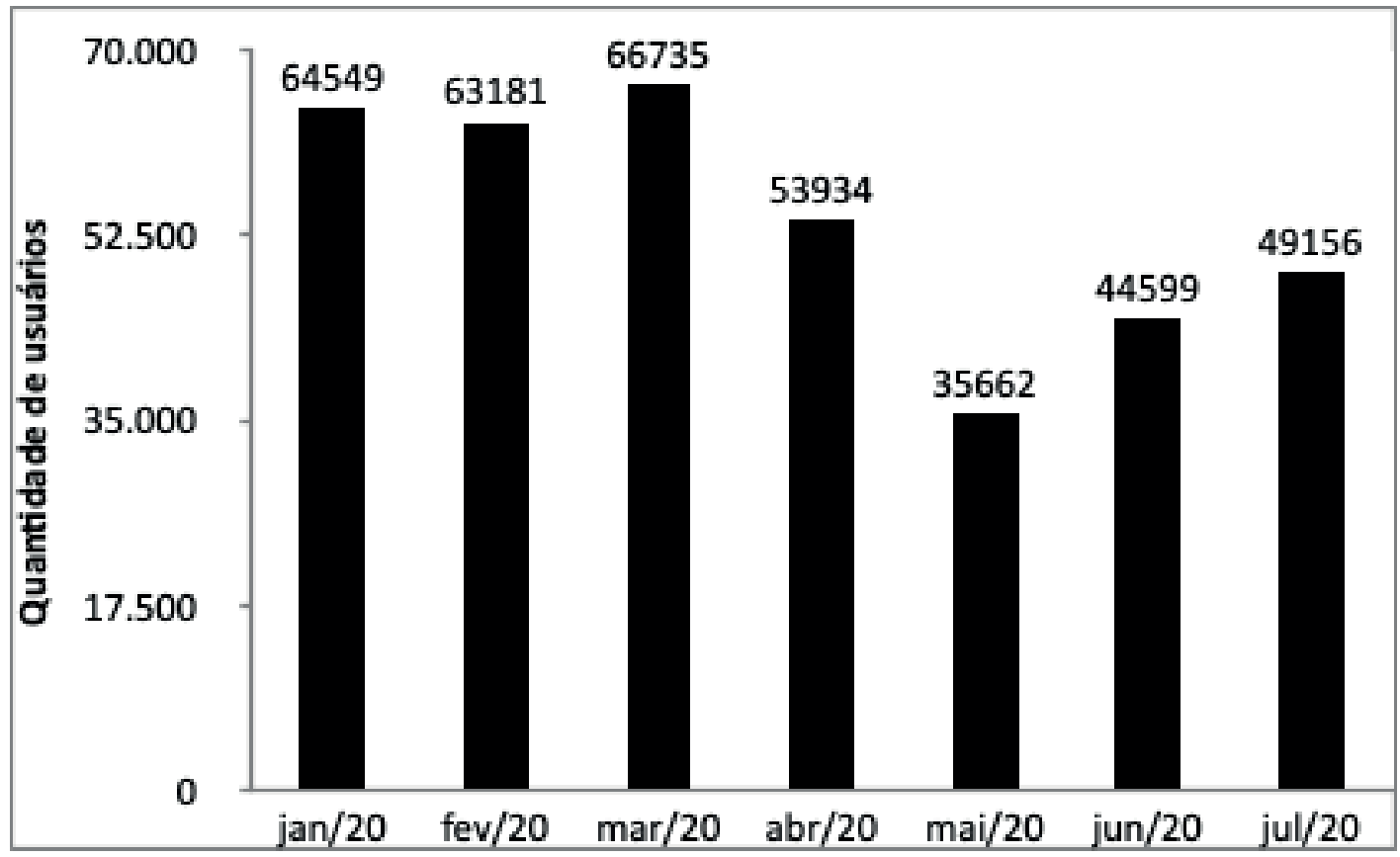

Fonte: Elaborado pelos autores (2020).

A média de atendimento mensal no serviço de dispensação de medicamentos no primeiro trimestre de 2020 foi de 64.822 usuários.

É possível verificar já no mês de abril uma redução do total de usuários atendidos. Nesse mês, que reflete o início da estratégia de entrega antecipada a partir da metade de março, houve redução de cerca de $17 \%$ no número de pacientes atendidos em relação à média do primeiro trimestre. $O$ mês de maio 
reflete a antecipação realizada em parte do mês de março e integralmente em abril, o que resultou em redução de cerca de $45 \%$ em comparação à média de usuários atendidos no primeiro trimestre. Os meses de junho e julho apresentaram redução de $31 \%$ e $24 \%$, respectivamente, e refletem as antecipações feitas nos meses anteriores. O retorno de pacientes cujas dispensações antecipadas ocorreram de março a abril contribuíram para a elevação do número de pacientes atendidos em junho e julho quando comparado ao mês de maio. Essa estratégia possibilitou a diminuição de 76 mil usuários circulando nas farmácias das Regionais de Saúde do Estado entre os meses de abril e julho/2020.

Para viabilizar a entrega de medicamentos para dois ou três meses de tratamento aos usuários, houve necessidade de reorganização das rotinas do CEMEPAR para aquisição e distribuição dos itens. Os processos de aquisição dos medicamentos destinados aos tratamentos ambulatoriais passaram a ter prioridade de tramitação na SESA/PR, para que fossem concluídos com mais agilidade e os medicamentos entregues no menor prazo possível, de modo a dar sustentabilidade à estratégia.

\section{c) Renovações automáticas para continuidade do tratamento}

Para a continuidade do tratamento no CEAF é necessário realizar, a cada três meses, sua renovação por meio da apresentação de um conjunto de documentos e exames de acordo com as exigências de cada PCDT. O usuário ou seu representante deve comparecer presencialmente à farmácia para entregar essa documentação.

Também no intuito de diminuir a circulação de pessoas nas farmácias e, além disso, considerando que muitos pacientes não tiveram acesso a consultas médicas ou à realização de exames, a SESA/PR implementou rotina de renovação automática dos tratamentos, por mais três meses, a todos os usuários, a partir de março/2020.

A cada mês, os usuários que tiveram seu tratamento renovado automaticamente receberam SMS com a seguinte mensagem "Para diminuir a circulação de 
pessoas, a FARMÁCIA DO PARANÁ RENOVOU AUTOMATICAMENTE o cadastro do seu MEDICAMENTO. NÃO venha à farmácia para renovação".

De março a julho de 2020 foram realizadas 338.666 renovações automáticas que viabilizaram a continuidade do tratamento sem que houvesse a necessidade de apresentação de documentação pelos pacientes. Essa estratégia também reduziu a circulação de pessoas e a presença delas nas farmácias.

\section{d) Desenvolvimento de ferramenta para cadastro online do usuário na farmácia}

A solicitação inicial de medicamentos por novos pacientes requer a sua ida ou a de seu representante até a farmácia para a entrega da documentação necessária para o cadastro.

Como alternativa ao cadastro presencial na farmácia, foi desenvolvido pela CELEPAR (Companhia de Tecnologia da Informação e Comunicação do Paraná), de forma emergencial, um formulário para pré-cadastro online do usuário com a possibilidade de envio de exames, laudos e demais documentos de forma eletrônica.

Este serviço, denominado "Pré-cadastro na Farmácia do Paraná"3, foi disponibilizado em abril de 2020, inicialmente aos usuários residentes nos municípios de Cascavel, Curitiba, Londrina e Maringá, por serem sede das Farmácias Regionais com maior volume de novas solicitações diárias. Para realizar o pré-cadastro, o paciente deve preencher um formulário e anexar os documentos necessários de acordo com medicamento e CID-10, podendo verificar essa documentação no serviço de "Consulta de Medicamentos da Farmácia do Paraná" disponível na plataforma PIÁ do Governo do Estado do Paraná4.

De 22 de abril a 29 de julho de 2020 foram realizados 400 pré-cadastros utilizando-se a ferramenta online.

3. O serviço de pré-cadastro está disponível no endereço <http://www.saude.pr.gov.br/medicamentos>.

4. O serviço de consulta de medicamentos está disponível no endereço <http://bit.ly/piamedicamentos>. 


\section{e) Ampliação do serviço “Remédio em casa”}

O serviço de entrega de medicamentos em casa foi instituído em 2017, no âmbito da SESA/PR, para os usuários cadastrados junto à Farmácia da 2a Regional de Saúde, residentes no município de Curitiba.

Com o objetivo de ampliar esse serviço e assim reduzir o número de pessoas que retiram presencialmente o medicamento na farmácia, foram selecionados os usuários que cumpriam os critérios definidos na Resolução SESA n 649/2019, que normatiza a entrega de medicamentos em domicílio.

Foi realizado contato telefônico com esses usuários para informá-los sobre a entrega do medicamento em sua residência e para confirmar seu endereço. Essa estratégia resultou num incremento de 1.745 usuários no serviço de entrega em casa da Farmácia da $2^{\text {a }}$ Regional, passando de 3.653 para 5.398 pacientes cadastrados.

Paralelamente, em abril de 2020, o serviço "Remédio em Casa" foi implantado na $10^{\text {a }}$ Regional de Saúde, para usuários residentes no município sede - Cascavel. Essa expansão foi possível em função da parceria estabelecida com a Secretaria Municipal de Saúde de Cascavel, que viabilizou a entrega em domicílio a 826 pacientes cadastrados nessa regional.

\section{ORIENTAÇÕES ÀS EQUIPES DE ASSISTÊNCIA FARMACÊUTICA NA SESA/PR}

No intuito de orientar as Farmácias e Centrais de Abastecimento Farmacêutico das 22 Regionais de Saúde, foram elaboradas e publicadas duas notas orientativas com informações e recomendações acerca das rotinas de trabalho e de enfrentamento à pandemia de Covid-19.

\section{Nota Orientativa às Farmácias}

A Nota Orientativa 05/202019, com recomendações para a reorganização dos 
processos de trabalho nas farmácias que dispensam medicamentos do Componente Especializado da Assistência Farmacêutica e do Elenco Complementar da SESA/PR frente à pandemia de Covid-19, foi publicada em 23/03/2020. Nela constam as orientações acerca de medidas preventivas de contágio, abertura de novos processos para solicitação de medicamentos, renovações e adequações dos tratamentos, dispensação de medicamentos e consultas farmacêuticas.

\section{Nota Orientativa às Centrais de Abastecimento Farmacêutico}

A Nota Orientativa $25 / 2020^{20}$, com recomendações para a reorganização dos processos de trabalho nas Centrais de Abastecimento Farmacêutico das Regionais de Saúde da SESA/PR frente à pandemia de Covid-19, foi publicada em 13/04/2020. Essa nota abordou medidas preventivas de contágio na distribuição de medicamentos, relacionadas principalmente a distanciamento social e medidas de higiene.

\section{CONSIDERAÇÕES FINAIS}

Esse relato de experiência demonstrou como foram planejadas e executadas as diferentes ações estratégicas adotadas no âmbito da Assistência Farmacêutica da SESA/PR no enfrentamento à Covid-19 e os resultados obtidos.

A organização da assistência farmacêutica na SESA/PR, que tem por base as diretrizes estabelecidas pela Coordenação de Assistência Farmacêutica em conjunto com o Centro de Medicamentos do Paraná, permitiu uma qualificada e tempestiva resposta ao enfrentamento da Covid-19, principalmente no sentido de reduzir o número de atendimentos nas farmácias das Regionais de Saúde sem comprometer, no entanto, a continuidade do tratamento do usuário.

As medidas foram efetivas na redução da circulação de pessoas e aumento do distanciamento social, diminuindo assim o risco de contágio pelo novo coronavírus tanto dos usuários quanto das equipes da assistência farmacêutica. 


\section{REFERÊNCIAS}

1. Organização Panamericana de Saúde. Folha informativa - COVID-19 (doença causada pelo novo coronavírus) [Internet]. Atualizada em 14 de julho de 2020 [citado em 2020 Jul 15]. Disponível em: https://www.paho.org.

2. Brasil. Portaria GM/MS n. 188, de 03 de fevereiro de 2020. Declara Emergência em Saúde Pública de importância Nacional (ESPIN) em decorrência da Infecção Humana pelo novo Coronavírus (2019-nCov) [Internet]. Diário Oficial da União; 2020. [citado em 2020 Jul 15]. ed. 24-A. Seção 1-Extra. Disponível em: http://www.in.gov.br.

3. Brasil. Lei n. 13.979, de 6 de fevereiro de 2020. Dispõe sobre as medidas para enfrentamento da emergência de saúde pública de importância internacional decorrente do coronavírus responsável pelo surto de 2019 [Internet]. Diário Oficial da União; 2020. [citado em 2020 Jul 15]. ed. 27; Seção 1. Disponível em: http://www.in.gov.br.

4. Ministério da Saúde [Internet]. Brasil confirma primeiro caso da doença. [citado em 2020 Jul 15]. Disponível em: https:// www.saude.gov.br.

5. World Health Organization. Coronavírus disease 2019 (COVID-19) situation report. Geneva: World Health Organization; 2020. 37.

6. Secretaria de Estado da Saúde do Paraná. Boletim - Informe Epidemiológico Coronavírus (COVID-19). 11 de março de 2020. [citado em 2020 Jul 29]. Disponível em http://www.saude.pr.gov.br.

7. Secretaria de Estado da Saúde [Internet]. Informação para a imprensa. [citado em 2020 Jul 27]. Disponível em http:// www.saude.pr.gov.br.

8. Paraná. Decreto n. ${ }^{4}$ 4.230, de 16 de março de 2020. Dispõe sobre as medidas para enfrentamento da emergência de saúde pública de importância internacional decorrente do Coronavírus - COVID-19 [Internet]. Diário Oficial Poder Executivo Paraná; 16 Mar 2020. [citado em 2020 Jul 25]. Disponível em: https://www.documentos.dioe.pr.gov.br.

9. Paraná. Decreto n. ${ }^{\circ}$ 4.298, de 19 de março de 2020. Declara situação de emergência em todo o território paranaense, nos termos do COBRADE n 1.5.1.1.0 - doenças infecciosas virais, para fins de prevenção e enfrentamento à COVID-19 [Internet]. Diário Oficial Poder Executivo Paraná; 19 Mar 2020. [citado em 2020 Jul 25]. Disponível em: https://www. documentos.dioe.pr.gov.br.

10. World Health Organization. Coronavírus disease 2019 (COVID-19) situation report. Geneva: World Health Organization; 2020 Mar 19. 59.

11. Secretaria de Estado da Saúde [Internet]. Casos em investigação Coronavírus (COVID-19). [citado em 2020 Jul 27 ]. Disponivel em http://www.saude.pr.gov.br.

12. Paraná. Decreto n.․ 4.319, de 08 de abril de 2020. Declara o estado de calamidade pública, como medida para enfrentamento da emergência de saúde pública de importância internacional decorrente do Coronavírus - COVID-19 [Internet]. Diário Oficial Poder Executivo Paraná; 08 abr 2020. [citado em 2020 Jul 25]. Disponível em: https://www. documentos.dioe.pr.gov.br.

13. World Health Organization. Coronavírus disease 2019 (COVID-19) situation report. Geneva: World Health Organization; 2020 Jul 28. 190

14. Secretaria de Estado da Saúde [Internet]. Informe Epidemiológico. 2020 Jul 28. [citado em 2020 Jul 29]. Disponível em http://www.saude.pr.gov.br.

15. Brasil. Ministério da Saúde (MS). Da excepcionalidade às linhas de cuidado: o Componente Especializado da Assistência Farmacêutica. Brasília: MS; 2010.

16. Ministério da Saúde (BR). Portaria de Consolidação nº 2 de 28 de setembro de 2017. Consolidação das normas sobre as políticas nacionais de saúde do Sistema Único de Saúde. Brasília: Diário Oficial da União; 2017. Poder Executivo, edição 192, seção 1.

17. Ministério da Saúde (BR). Portaria de Consolidação nº 6 de 28 de setembro de 2017. Consolidação das normas sobre o financiamento e a transferência dos recursos federais para as ações e os serviços de saúde do Sistema Único de Saúde. Brasília: Diário Oficial da União; 2017. Poder Executivo, edição 190, Seção 1.

18. Secretaria de Estado da Saúde do Paraná. Plano Estadual de Saúde do Paraná 2016-2019 [Internet]. Curitiba: SESA; 2016. Disponível em: http://www.saude.pr.gov.br.

19. Secretaria de Estado da Saúde do Paraná [Internet]. Nota Orientativa 05/2020 - Recomendações para a reorganização dos processos de trabalho nas farmácias que dispensam medicamentos do componente especializado da assistência 
farmacêutica e do elenco complementar da SESA-PR frente à pandemia de covid-19. Atualizada em 24 de março de 2020. [citado em 2020 Jul 30]. Disponível em: http://www.saude.pr.gov.br.

20. Secretaria de Estado da Saúde do Paraná [Internet]. Nota Orientativa 42/2020 - Recomendações para a reorganização dos processos de trabalho nas centrais de abastecimento farmacêutico das regionais de saúde da SESA/PR frente à pandemia de covid-19. Atualizada em 13 de abril de 2020. [citado em 2020 Jul 30]. Disponível em: http://www.saude. pr.gov.br.

RECEBIDO:31/07/2020

ACEITO: 07/10/2020 